\title{
Transfemoral Aortic Valve Replacement (TAVR): Is Incorporation of Interventional Radiologists into the Team Beneficial?
}

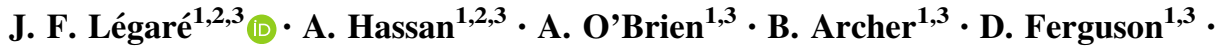

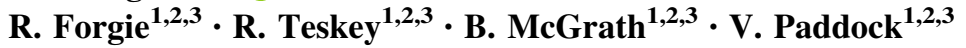

Received: 6 August 2019/Revised: 14 August 2019/Accepted: 16 August 2019/Published online: 30 August 2019 (C) Springer Science+Business Media, LLC, part of Springer Nature and the Cardiovascular and Interventional Radiological Society of Europe (CIRSE) 2019

\section{Editorial}

The modern treatment of aortic valve disease is rapidly changing with significant emphasis on collaborative approaches to the decision-making around cardiac care. The Heart Team model of care has therefore been endorsed by major cardiological societies to be the standard of care, but to date [1] there are no clear guidelines on its composition. This is particularly important if one where to consider that vascular access has evolved as a critical component for transcatheter aortic valve replacement (TAVR) feasibility and success. It is currently accepted that the best outcomes are derived from TAVR when performed transfemorally when compared to alternate access sites [2]. Despite excellent outcomes in TAVR, there remains significant risk of complications, in particular related to the vascular access with more than $30 \%$ of patients with significant peripheral vascular disease (PVD) and vascular complications often reported in more than $10 \%$ of patients [3].

Given the importance of vascular access, we believe that interventional radiologists bring a unique set of expertise to a TAVR Heart Team based on their expertise in peripheral vascular disease therapies. This explains why at the New Brunswick Heart Centre (NBHC) our TAVR program has always included interventional radiology, interventional cardiology, and cardiac surgery as team members. Since 2010, more than 400 TAVR procedures have been performed with excellent outcomes and an overall mortality rate of $1.5 \%$. During that time, our TAVR program has evolved significantly by adopting increasingly less invasive approach to patients undergoing TAVR as demonstrated by our adoption of percutaneous approach and conscious sedation. There are to the best of our knowledge few centers where interventional radiology plays such a critical role in the Heart Team. Their expertise has been essential in our gradual transition to a $100 \%$ percutaneous transfemoral approach. This is in spite of $35 \%$ of patients with significant known PVD and a significant proportion having small access vessels $(26 \% \leq 7 \mathrm{~mm})$. We also reported a $1.2 \%$ major vascular complication rate, which compares favorably to other large series [4].

Interventional radiologists have in many institutions been key clinicians involved in the management of peripheral vascular disease. This explains at least in part why interventional radiologists have proved to be invaluable members of our

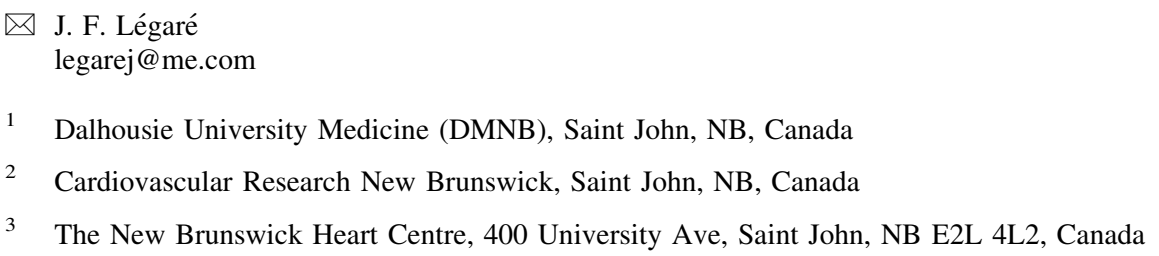


TAVR team. Overall, we have developed a system in which TAVR procedures are performed in a collaborative manner and where all team members are valued. Critical decisions have not relied on a single individual but instead on overall consensus. We argue that the New Brunswick Heart Centre TAVR Team is an example of a well-functioning "Heart Team" and stands in contrast to programs in which outcomes are highly dependent on the team leader rather than a shared responsibility model. Our shared responsibility model promotes respect and understanding of the extensive experience and complementary skills of each member. We believe that our team composition will be particularly critical as TAVR indications are expanding into lower risk patients in which the margin for error or complications will be lower. There are important qualities and experience that can be gained from interventional radiologist who does peripheral vascular interventions as part of their daily work, which is difficult to measure or quantify. It is important to note that this does not mean that all centers should adopt our approach or that interventional radiology should become the standard of care, but it does point out key features of teamwork that are increasingly being recognized and have been difficult to define and implement in medicine [5].

Funding This study was not supported by any funding.

\section{Compliance with Ethical Standards}

Conflict of interest The authors declare that they have no conflict of interest. All authors state that they have not had financial support or relationships that may pose any conflict of interest in the present manuscript.

\section{References}

1. Antonides CFJ, Mack MJ, Kappetein AP. Approaches to the role of the heart team in therapeutic decision making for heart valve disease. Struct Heart. 2017;1(5-6):249-55.

2. Himbert D, et al. Results of transfemoral or transapical aortic valve implantation following a uniform assessment in high-risk patients with aortic stenosis. J Am Coll Cardiol. 2009;54(4):303-11.

3. Sinning JM, et al. The impact of peripheral arterial disease on early outcome after transcatheter aortic valve implantation: results from the German Transcatheter Aortic Valve Interventions Registry. Am Heart J. 2012;164(1):102-10.e1.

4. Malyar NM, et al. Prevalence and impact of critical limb ischaemia on in-hospital outcome in transcatheter aortic valve implantation in Germany. EuroIntervention. 2017;13(11):1281-7.

5. Rosenbaum L. Divided we fall. N Engl J Med. 2019;380(7):684-8.

Publisher's Note Springer Nature remains neutral with regard to jurisdictional claims in published maps and institutional affiliations. 